\title{
Aneurisma de arteria renal. Nefrectomía laparoscópica, reconstrucción en banco y autotrasplante
}

\author{
Blanco Díez A, Armas Molina J, Alvarado Rodríguez A, Alcaraz Asensio A*, Artíles Hernández J, \\ Chesa Ponce N.
}

\author{
Servicio Urología. Hospital Universitario Insular de Gran Canaria. *Servicio Urología. \\ Hospital Clínic i Provincial. Barcelona.
}

Actas Urol Esp. 2008;32(7):763-766

\section{RESUMEN}

\section{ANEURISMA DE ARTERIA RENAL. NEFRECTOMÍA LAPAROSCÓPICA, RECONSTRUCCIÓN EN BANCO Y} AUTOTRASPLANTE

Se presenta un caso de paciente hipertenso severo con mala respuesta a pesar de 5 fármacos, el cual es diagnosticado de aneurisma en arteria renal derecha, durante el estudio de su HTA. Se realiza cirugía conservadora renal haciendo extracción laparoscópica del injerto, reconstrucción en banco del pedículo, y posterior trasplante en fosa ilíaca derecha aprovechado la incisión ilioinguinal empleada para la extracción, sin necesidad de dos incisiones. Se realiza breve discusión de indicaciones de la cirugía en estos pacientes.

Palabras clave: Aneurisma de arteria renal. Cirugía. Hipertensión arterial.

\section{ABSTRACT \\ RENAL ARTERY ANEURYSM. LAPAROSCOPIC NEPHRECTOMY, EX-VIVO RECONSTRUCTION AND AUTOTRASPLANTATION}

We report a case of severe hypertensive patient with poor response regardless 5 drugs, that is diagnosed with right renal artery aneurysm, during the study of his HTN. Conservative surgery was performed by extracting laparoscopic kidney graft,ex-vivo pedicle reconstruction, followed by transplant in right iliac fossa utilizing the ilioinguinal incision used for the extraction, without need for two incisions. We perform a brief discussion of surgery indications of surgery in these patients.

Keywords: Renal artery aneurysm. Surgery. Hypertension.

$\mathrm{L}$ os aneurismas de arteria renal son entidades poco frecuentes, que sin embargo serán cada vez más diagnosticados debido al uso creciente de técnicas de imagen. Presentamos un caso clínico de aneurisma de arteria renal derecha que es resecado en banco y el riñón autotrasplantado. Nos parece de interés que la nefrectomía se hizo por vía laparoscópica, extrayendo la pieza por la misma incisión a través de la cual se va a autotrasplantar, lo que nos ha permitido minimizar la agresión quirúrgica $\mathrm{y}$ ofrecer al paciente una sola incisión.

\section{CASO CLÍNICO}

Paciente varón de 48 años. Diabetes Mellitus tipo II de 7 años de evolución. Tabaquismo de 40 ci- garrillos/día. Episodios de angina con varios ingresos en cardiología. Dislipemia. Tensión arterial no controlada a pesar de múltiple terapia con 5 fármacos: Amlodipino $10 \mathrm{mg}$, Torasemida $5 \mathrm{mg}$, Carvedilol $25 \mathrm{mg}$, Doxazosina $8 \mathrm{mg}$, y Olmesartán 40 mg.

En su estudio diagnóstico de HTA renovascular se realiza RX y ecografía abdominal que son normales. El renograma test de Captopril refiere baja probabilidad de hipertensión renovascular. Se realiza Arteriografía renal objetivándose aneurisma de $2,5 \mathrm{~cm}$ en bifurcación de arteria renal principal derecha (Fig. 1).

Con dicho diagnóstico se plantea al paciente, nefrectomía derecha laparoscópica con reconstrucción del pedículo renal y aneurismectomía en banco y posterior autotrasplante en fosa ilíaca derecha. 


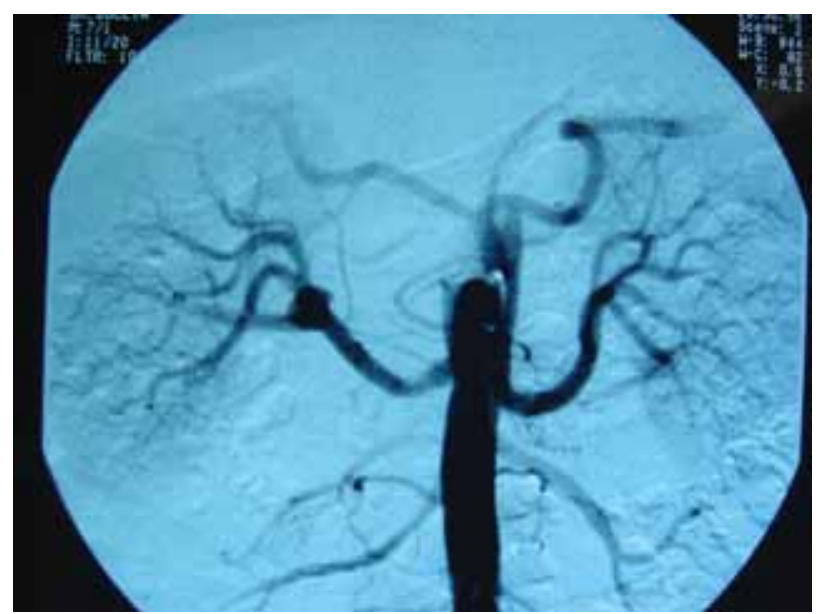

FIGURA 1. Aneurisma de arteria renal en arteriografia.

\section{Cirugia}

Extracción laparoscópica

Decúbito lateral izquierdo. Se colocan 5 trócares en triangulación. Apertura caudal del parietocólico derecho localizando uréter que se secciona a la altura del cruce ilíaco. Disección de cara anterior de cava. Localización y disección de vena renal derecha. Acceso y liberación a valva posterior renal dejando el órgano adherido a pared en polo superior. Disección de arteria renal hasta su localización retrocava para obtener la máxima longitud. Se libera con Ligasure el polo superior conservando la suprarrenal y dejando el riñón únicamente colgado por su pedículo vascular. En este momento se realiza incisión ilioinguinal derecha de 6 centímetros a través de la cual se introduce la mano izquierda del cirujano para controlar el pedículo. Clip Hemolock en arteria en porción retrocava, y sección de la misma. Idem en vena. Se extrae la pieza.

\section{Cirugía de banco}

Tiempo de isquemia caliente (3 minutos). Se comienza la infusión por arteria de solución Eurocollins, y enfriamiento renal con compresas húmedas y hielo sobre ellas. Se limpia el injerto de su grasa y se diseca el uréter hasta $5 \mathrm{~cm}$ del hilio, para preservar su vasculatura y evitar complicaciones posteriores (fístulas-estenosis).

Se diseca arteria renal principal hasta aneurisma y se disecan arterias que emergen del mismo. Disposición: se observa aneurisma de $2,5 \mathrm{~cm}$ en la bifurcación de arteria renal principal y del que emergen dos arterias renales, existiendo una tercera que emerge de pared arterial sana (pero a la misma altura que las otras dos). Existe una arteria polar preaneurismática que se conserva.

Se diseca el aneurisma sacular, de base amplia, que afecta al $90 \%$ de la circunferencia arterial, hasta la base del mismo. Se secciona todo el aneurisma a través de su base siguiendo la circunferencia y quedando el pedículo abierto en Y con una de las tres ramas manteniendo su circunferencia y su parche pero las otras dos quedando abiertas en libro. Se realiza sutura continua en pantalón con Prolene 7/0 en ambas caras de la Y resecadas para recuperar la anatomía. Se diseca la vena que aparece separada del aneurisma, hasta su entrada en el hilio renal, dando una longitud de unos $3 \mathrm{~cm}$. Se perfunde el riñón objetivándose estanqueidad de suturas.

\section{Trasplante renal}

Con el paciente en decúbito supino y sobre la misma incisión ampliada que se realizó para extraer la pieza (ilioinguinal de Gibson), se realiza el trasplante. Se efectúan las siguientes anastomosis: Vena renal sobre vena ilíaca externa término-lateral con sutura continua en cada cara de prolene 5/0. Arteria renal (con boca seccionada en bisel) sobre arteria ilíaca externa término-lateral con sutura contínua de $360^{\circ}$ Prolene 5/0. Tras desclampaje se observa diuresis espontánea a los 5 minutos, excelente coloración y consistencia del injerto, con buen thrill arterial y buen relleno venoso. Se realiza implante ureteral según técnica de Politano-Leadbetter sin dejar Doble J. dejamos drenaje Jackson-Pratt y cerramos en tres planos el lecho quirúrgico. Se observa una única incisión. (Fig. 2).

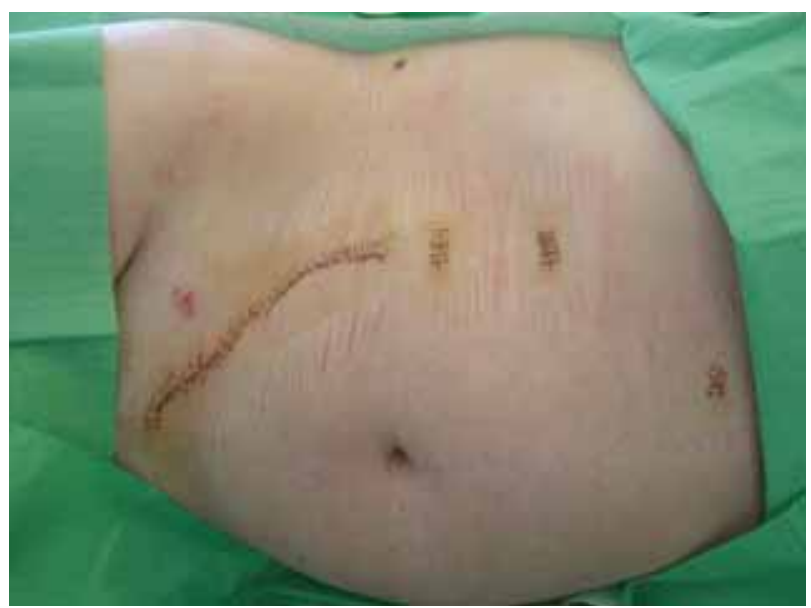

FIGURA 2. Incisión única operatoria. 


\section{Evolución}

La evolución es óptima siendo alta al $7^{\circ}$ día postintervención. El $4^{\circ}$ día se realiza un eco-doppler del injerto que muestra índices de resistencia en ramas periféricas normales, con ligero aumento de las resistencias en ramas proximales. Para valorar la función renal se efectúa también el $4^{\circ}$ día un renograma diurético MAG 3 que objetiva una perfusión homogénea del injerto, con buena capacidad de concentración, tiempo de tránsito intraparenquimatoso adecuado, y retención del trazador en sistema pielocalicial con adecuada respuesta al estímulo diurético. Figura 3. El Angio Tac a los 6 meses muestra ausencia de aneurisma arterial y anastomosis reno-ilíaca con ostium amplio sin estenosis. (Fig. 4). El paciente está normotenso (120/70), con dos fármacos.

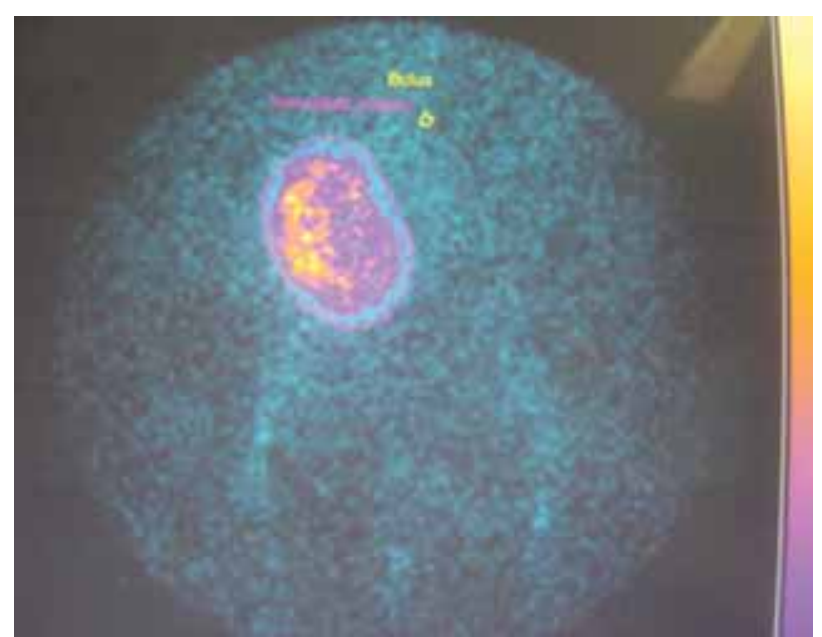

FIGURA 3. Renograma post transplante.

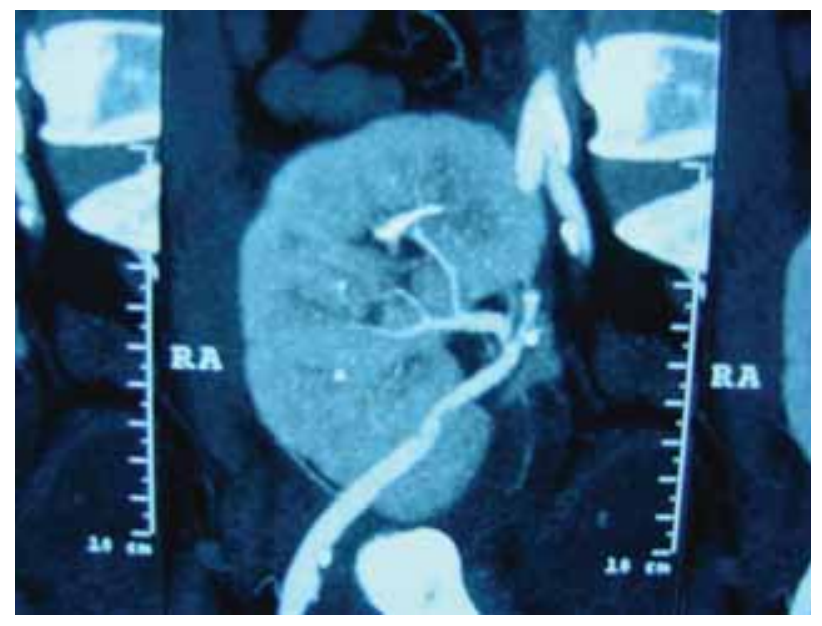

FIGURA 4: Angio-TAC a los 6 meses.

\section{DISCUSIÓN}

Los aneurismas de la arteria renal son poco comunes, desconociéndose su prevalencia. Se diagnostican con una incidencia de $0.7-1.3 \%$ en las angiografías renales. La etiología suele estar en relación con displasia fibromuscular la arteriosclerosis de la arteria renal, pudiendo ser congénitos, asociados con arteritis, o con antecedente traumático. Causas menos comunes son la sífilis o la tuberculosis. Habitualmente son asintomáticos, sin embargo en algún caso pueden producir dolor en flanco (15\%), hematuria (30\%), hipertensión (55\%), etc ${ }^{1-4}$.

Existen cuatro tipos:

- Aneurismas saculares: los más comunes (93\% de todos los aneurismas) usualmente en la bifurcación de la arteria renal principal, y asociados a arteriosclerosis o fibrodisplasia. Pueden causar erosión de vena renal, de la pelvis renal, o trombosis.

- Aneurismas fusiformes: aparecen en jóvenes hipertensos con fibrodisplasia. La complicación más importante es la trombosis de la arteria renal.

- Aneurismas disecantes: por disección de la elástica interna.

- Aneurismas intrarrenales: causados por arteriosclerosis, trauma, malformaciones congénitas vasculares, displasia fibrosa, o biopsias renales con aguja.

Existen varias complicaciones asociadas a esta patología, como son: trombosis de arteria renal o embolización distal, hematuria por ruptura del aneurisma dentro del sistema colector, infarto renal por disección aneurismática, o embolismo distal. Por último hidronefrosis por compresión del sistema colector

A pesar de la existencia de un $70 \%$ de incidencia de hipertensión en aneurismas de arteria renal, no se ha establecido una clara relación causal. Existe la teoría de que se trata de una hipertensión mediada por renina, secundaria a una isquemia relativa del parénquima renal ${ }^{1,6}$. La mayoría de los aneurismas se han hallado durante el estudio de una hipertensión renovascular, por tanto, un número elevado de pacientes con aneurismas de arteria renal tienen hipertensión.

La rotura aneurismática es rara pero es una complicación letal. Los aneurismas extrarrenales, o incompletamente calcificados en su circunferencia, 
son más proclives a la ruptura que aquellos con calcificación circunferencial. La ruptura de dichos aneurismas durante el embarazo es una complicación catastrófica con una mortalidad del 50\% para la madre y del $78 \%$ para el feto.

La Urografía Intravenosa, sugiriendo lesión vascular se observa en el $60 \%$ de casos, sin embargo las pruebas en las que se detecta más frecuentemente es en la ecografía y el T.A.C. Ante hallazgos sugestivos se debe realizar siempre un Angiografía que es la prueba fundamental de estudio.

Las indicaciones quirúrgicas son: 1) Hipertensión no controlada, 2) Calcificación anular incompleta, 3) Tamaño del aneurisma $>2-2,5 \mathrm{~cm}$ (según autores), 4) Mujer en edad fértil, 5) Aumento progresivo de tamaño en controles sucesivos, 6) Fístula arteriovenosa, 7) Complicaciones relacionadas: hematuria, infarto renal, ruptura, etc. En el resto de lo paciente puede ser manejado de forma conservadora $^{1,3,4}$. Existen otros autores que sugieren la reparación en todos los casos independientemente del tamaño y de los síntomas para evitar los microembolismos en el parénquima renal, o el desarrollo de hipertensión mantenida ${ }^{5}$. En el sentido opuesto otros autores refieren que los pacientes asintomáticos, independientemente de otras variables pueden ser vigilados sin que por ello aumente el número de potenciales complicaciones ${ }^{6}$.

Las opciones quirúrgicas incluyen: embolización ${ }^{1,5}$, resección del aneurisma y anastomosis directa, resección y reparación con parche de Dacron, autotransplante tras resección del aneurisma, reconstrucción ex-vivo para realizar anastomosis múltiples $^{1,4,7}$, nefrectomía parcial, y nefrectomía. En algún caso se podría colocar un stent intraaneurismático por vía percutánea ${ }^{8}$. Mención aparte merecen las nuevas tecnologías para reparar aneurismas sin necesida de realizarlo ex vivo, como son la cirugía laparoscópica pura y la cirugía robótica ${ }^{9-11}$. Pensamos que estos son los caminos del futuro, ya presente en algunos lugares.

\section{REFERENCIAS}

1. Walsh, P.C.; Retik, A.B.; Vaughan, E.D.; Wein, A.J. Campbell's Urology Eighth Edition. Ed Saunders Company. Phliadelphia. 2002.

2. Poutasse EF. Renal artery aneurisms. J Urol. 1975;113(4):443449.

3. Lumsden AB, Salam TA, Walton KG. Renal artery aneurisms: a report of 28 cases. Cardiovasc Surg. 1996;4(2):185-189.

4. Bulbul MA, Farrow GA. Renal artery aneurisms. Urology. 1992; 40(2): 124-126.

5. Hupp T, Allenberg JR, Post K, Roeren T, Meier M, Clorius JH. Renal artery aneurisms: surgical indications and results. Eur $\mathrm{J}$ Vasc Surg. 1992;6(5):477-486.

6. Hubert JP, Pairolero PC, Kazmier FJ. Solitary renal artery aneurism. Surgery. 1980;88(4):557-565.

7. Dubernard JM, Martin X, Gelet A, Mongin D. Aneurysms of the renal artery: surgical management with special reference to extracorporeal surgery and autotransplantation. Eur Urol. 1985; 11(1):26-30.

8. Liguori G, Trombetta C, Bucci S, Pozzi-Mucelli F, Bernobich E, Belgrano E. Percutaneous management of renal artery aneurism with a stent graft. J Urol. 2002;167(6):2518-2519.

9. Castillo OA, Peacock L, Diaz M, Orellana S, Urena RD. Case report: laparoscopic repair of saccular renal-artery aneurysm. J Endourol. 2006;20(4):260-261.

10. Luke P, Knudsen BE, Nguan CY, Pautler SE, Swinnimer S, Kiaii $\mathrm{R}$, et al. Robot-assisted laparoscopic renal artery aneurysm reconstruction. J Vasc Surg. 2006;44(3):651-653.

11. Gill IS, Murphy DP, Hsu TH, Fergany A, El Fettouh H, Meraney AM. Laparoscopic repair of renal artery aneurysm. J Urol. 2001; 166(1):202-205

Correspondencia autor: Dr. A. Blanco Díaz

Servicio de Urología

Hospital Universitario Insular de Gran Canaria.

Plaza del Doctor Pasteur, s/n. 35016 - Las Palmas de Gran

Canaria. Tel.: 928444000

E-mail autor: abdiez2003@yahoo.es

Información artículo: Nota Clínica

Trabajo recibido: julio 2007

Trabajo aceptado: septiembre 2007 